\title{
Establishing traceability of scatterometry to the SI meter
}

\author{
P. E. Hansen and L. Nielsen \\ Danish Fundamental Metrology, Technical University of Denmark, DK-2800 Kgs. Lyngby, Denmark
}

\begin{abstract}
We present an uncertainty estimate for numerical reconstruction of grating profiles using scatterometry and show how traceability to the SI meter may be obtained using measurement standards. By describing the propagation of light through non-ideal optical components and the light interaction with a nonideal sample under test, we establish a model function for the detected power that contains all the significant error sources in the setup. After having assigned typical uncertainties to the parameters of the optical elements, the model function is used to investigate the effect of sample misalignment for varying angle of incidents. This study shows that normal incident is preferable with respect to uncertainties. We continue by considering measurements performed with non-ideal settings and investigate how these erroneously settings affect the scatterometry parameters of the sample, such as height, fill factor and wall angles found from a least squares match between measured and calculated diffraction efficiencies. The input to this least squares method is 1) best estimates and the associated covariance matrix of all measured quantities, including influence quantities, and 2) a measurement model describing all known physical relations between the measured quantities and the scatterometry parameters. The output includes best estimates and the associated covariance matrix of the scatterometry parameters. Having the model function for the measurement, the uncertainties of all relevant quantities and a robust least squares algorithm, we can establish a traceability chain to the SI meter.
\end{abstract}

This is an Open Access article distributed under the terms of the Creative Commons Attribution-Noncommercial License 3.0, which permits unrestricted use, distribution, and reproduction in any noncommercial medium, provided the original work is properly cited. 